\title{
Search for physics beyond the SM in meson decays with WASA detector.
}

\author{
Damian Pszczel ${ }^{* \dagger}$ \\ Uppsala University \\ National Centre for Nuclear Research \\ E-mail: damian.pszczelephysics.uu.se
}

The indirect searches for dark matter particles are a very hot topic of today's physics and astrophysics. The energy-mass content of the Universe is one of the biggest riddles of modern science. The Standard Model describes the physics of only a small fraction of the Universe. Although only gravitational interaction of the dark matter with normal matter was observed up to now, a lot of effort is put by the scientific community into searches for some new interaction between dark and usual matter. This new force would be carried by a new boson and the latter could be seen in the decays of mesons. Most of the scientific research for the effects of dark matter focuses on very high energy physics, still the existence of such effects at lower energy scales is not excluded. Through the analysis of the rare decays of light mesons (eta, neutral pion) WASA collaboration searches for a dark matter signal which cannot be described in the frame of the Standard Model. This effect could be explained by a coupling between a dark boson and Standard Model particles (leptons, photons and/or quarks). The WASA detector is perfectly suited to study leptonic decays for it has the capability to detect both neutral and charged particles and particle identification capacity. We will present a short description of the analysis of meson decay channels $\pi^{0} \rightarrow e^{+} e \gamma$, $\eta \rightarrow e^{+} e \gamma, \eta \rightarrow e^{+} e$ and $\eta \rightarrow \pi^{0} e^{+} e^{-}$. The data sample with $\eta$ meson production was collected in proton proton collisions at $1.4 \mathrm{GeV}$ kinetic energy.

The European Physical Society Conference on High Energy Physics

5-12 July

Venice, Italy

* Speaker.

${ }^{\dagger}$ Supported by the Polish National Science Centre grant no.UMO-2014/15/N/ST2/03179. 


\section{Motivation for physics beyond the Standard Model.}

It is undeniable that the search for a dark energy-mass content of the Universe is one of the most fundamental topics of the modern day physics. The scientific community makes an enormous effort in order to design and run experiments sensitive enough to catch new particles that interact extremely weakly with Standard Model matter but that could explain the gravitational effects we observe while, for example, looking at the rotational velocities of galaxies or reconstructing the large scale structure formations. While most of the scientific research for signatures of dark matter focuses on very high energy physics, still the existence of such effects at lower energy scales is not excluded.

Dark particles interact weakly with the usual matter through one or more mediators that are coupled to the SM via a portal. There are different types of portals depending on the mediator spin and parity - scalar, pseudoscalar, fermion or vector. Some astrophysics observations such as the positron and/or electron excesses observed by PAMELA [1], ATIC [3], H.E.S.S. [2] and AMS [5] as well as the narrow $0.511 \mathrm{MeV} \gamma$ ray emission from the galactic bulge observed by INTEGRAL [4] may indicate the presence of new undiscovered particles that decay to $e^{+} e^{-}$pairs. Those measurements are our principal motivation to focus on the hypothetical vector portal and its associated vector mediator.

The hypothesis of a new boson solving the problem of the dark matter content in the Universe is an idea that emerged in the 80 's. Multiple authors postulated an extra $U(1)_{\text {dark }}$ abelian gauge field and the associated light vector boson, also called the dark photon, in the $\mathscr{O} \mathrm{MeV}-\mathrm{GeV}$ mass range, as a possible extension of the SM $[6,7,8]$.

The dark photon is a vector field $A_{\mu}^{\prime}$ with Lagrangian:

$$
\mathscr{L}_{A^{\prime}}=-\frac{1}{4} F^{\prime \mu v} F_{\mu v}^{\prime}+\frac{\varepsilon}{2 \cos \theta_{W}} B^{\mu v} F_{\mu v}^{\prime}-\frac{1}{2} m_{A^{\prime}}^{2} A^{\prime \mu} A_{\mu}^{\prime}
$$

where $F_{\mu \nu}^{\prime} \equiv \partial_{\mu} A_{v}^{\prime}-\partial_{v} A_{\mu}^{\prime}$ is the dark photon field strength and $B_{\mu \nu} \equiv \partial_{\mu} B_{v}-\partial_{v} B_{\mu}$ is the SM hypercharge field strength. This model of the minimal kinetically mixed dark photon ([9]) is parametrized by the dark photon mass $m_{A^{\prime}}$ and the kinetic mixing parameter $\varepsilon$. For $m_{A^{\prime}}$ in the range of $\mathrm{MeV}-\mathrm{GeV}$, the dominant effect of this kinetic mixing (after electroweak symmetry breaking) is an analogous mixing with the SM electromagnetic field strength $F^{\mu v}$ expressed by $\frac{\varepsilon}{2} F_{\mu v}^{\prime} F^{\mu v}$. The result of this mixing is that the dark photon acquires a coupling of strength $e \varepsilon$ to the electromagnetic current.

\section{Search for a dark photon in meson decays with WASA-at-COSY.}

\subsection{The $\pi^{0} \rightarrow e^{+} e^{-} \gamma$ channel.}

Using the data collected by the WASA-at-COSY experiment two searches for a dark photon were performed. The first result, published in [10], is based on the $\pi^{0} \rightarrow e^{+} e^{-} \gamma$ decay (also called Dalitz decay). In this experiment, the $\pi^{0}$ were produced in proton-proton collisions at a kinetic beam energy of $550 \mathrm{MeV}$.

The idea of the analysis was to search for narrow structure in the invariant mass spectrum of $e^{+} e^{-}$pair (see figure 1) for dark photon mass in 20-100 MeV/c $c^{2}$ range (less than twice the muon 


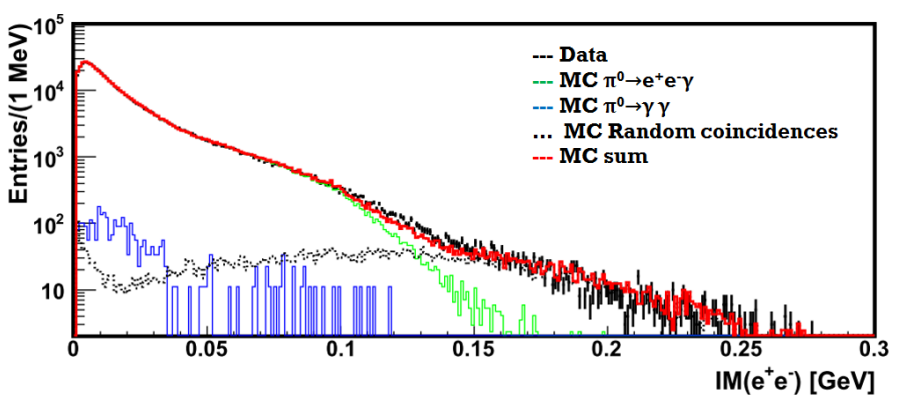

Figure 1: $\pi^{0} \rightarrow e^{+} e^{-} \gamma$ analysis: invariant mass of $e^{+} e^{-}$ (from [10]).

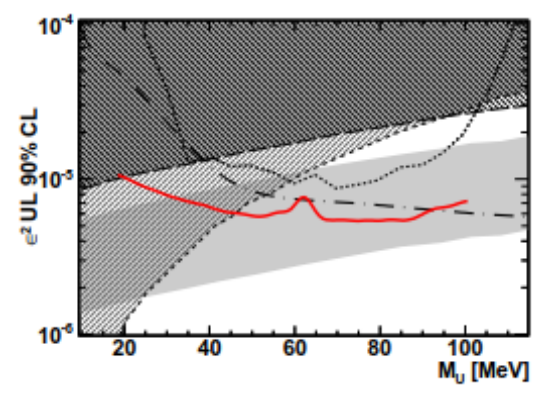

Figure 2: $\varepsilon^{2}$ upper limit as a function of the $e^{+} e^{-}$invariant mass (from [10]).

mass). For $\varepsilon^{2}>10^{-6}$ the average path traveled by a dark photon emitted in low energy $\pi^{0}$ decay should be less than a millimeter, therefore we are, in principle, able to detect its decay inside our detector.

This analysis led to the estimation of the upper limit on the coupling parameter $\varepsilon^{2}$ shown in figure 2 .

\subsection{The $\eta \rightarrow e^{+} e^{-} \gamma$ channel.}

The procedure developed for the dark photon search in $\pi^{0} \rightarrow e^{+} e^{-} \gamma$ channel is naturally extended to the $\eta \rightarrow e^{+} e^{-} \gamma$ channel. We have data collected in 2012 in proton-proton collisions at 1.4 $\mathrm{GeV}$ kinetic beam energy. The advantage with respect to the former analysis is that we can cover the invariant mass spectrum up to the $\eta$ meson mass $\sim 548 \mathrm{MeV} / \mathrm{c}^{2}$ while the pion mass limits the accessible phase space to $\sim 135 \mathrm{MeV} / \mathrm{c}^{2}$.

The figure 3 shows a selected data sample and Monte Carlo simulations of background channels. The difference between data and simulations is plotted in figure 4. We see no peak structure and can therefore set an upper limit on $\varepsilon^{2}$. The result will be released soon.

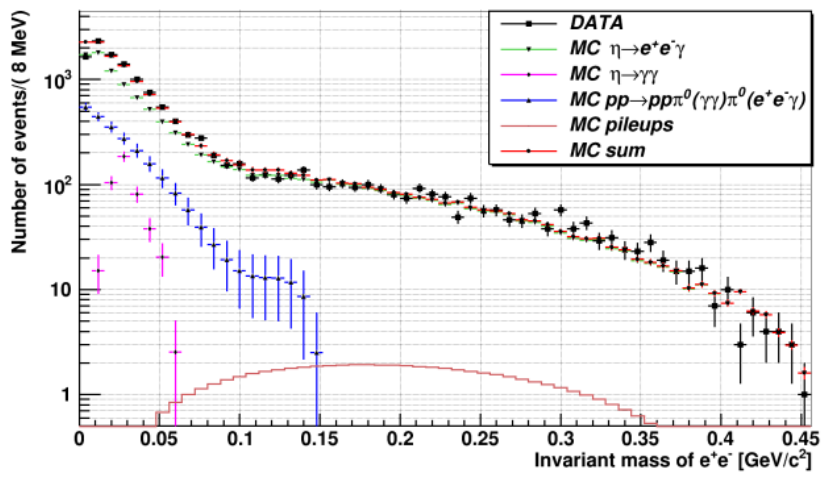

Figure 3: $\eta \rightarrow e^{+} e^{-} \gamma$ analysis: invariant mass of $e^{+} e^{-}$.

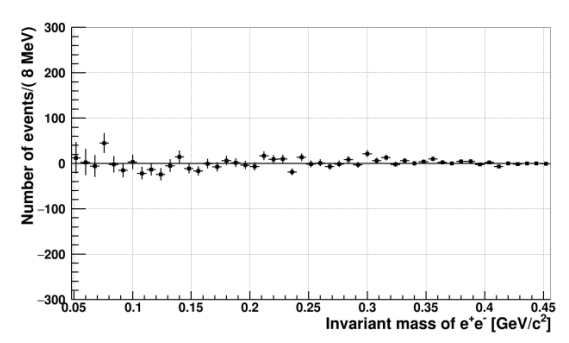

Figure 4: Difference between data and simulations: invariant mass of $e^{+} e^{-}$.

\section{3. $\eta$ meson transition form factor extraction.}

The selected data sample of $\eta \rightarrow e^{+} e^{-} \gamma$ event candidates can also be used to extract the transi- 
tion form factor of the $\eta$ meson. This function of the invariant mass of $e^{+} e^{-}$contains information about the inner structure (quark and gluon) of a particle. The existence of non-Standard Model particles could influence the value of the form factor. Equation 3.1 shows that the form factor is unity for a point-like particle.

$$
\frac{d \sigma}{d q^{2}}=\left|\frac{d \sigma}{d q^{2}}\right|_{Q E D}\left|F\left(q^{2}\right)\right|^{2}
$$

An insightful introduction to this concept can be find in [11], here we just recall that in the Vector Meson Dominance model, the form factor function is approximated by:

$$
F\left(q^{2}\right)=\sum_{V} \frac{M_{V}^{2}}{M_{V}^{2}-q^{2}-i M_{V} \Gamma_{V}\left(q^{2}\right)} \cong \frac{1}{1-\frac{q^{2}}{M_{V}^{2}}}
$$

where $V=\rho, \omega, \phi^{1}, \Gamma_{V}\left(q^{2}\right)$ is the total width and $M_{V}$ the mass of the vector meson, $q^{2}$ is the exchanged four-momentum squared, here equal to the invariant mass of $e^{+} e^{-}$.

The equation 3.2 is often used as a fit function. The $M_{V}$ vector meson mass is replaced by a free parameter $\Lambda$ and, for convenience, the value of $\Lambda^{-2}$ (sometimes called $b_{\eta}$ ) is provided as result of form factor calculations.

The figure 5 represents the result of dividing data by the Monte Carlo simulations in different bins of $e^{+} e^{-}$invariant mass. We use the equation 3.2 with one free parameter $\Lambda^{-2}$ to fit the distribution.

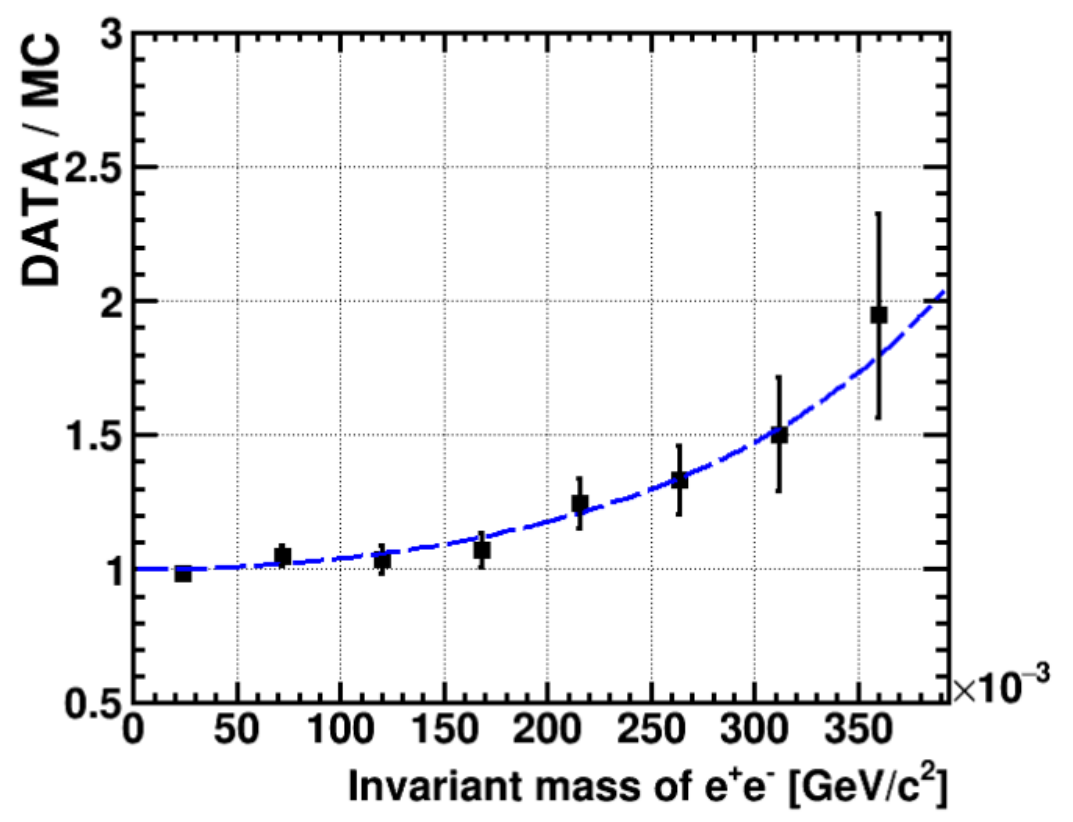

Figure 5: $\eta \rightarrow e^{+} e^{-} \gamma$ analysis: $\eta$ form factor fit.

This fit leads to the form factor value of $\Lambda^{-2}=(1.96 \pm 0.29) \mathrm{GeV}^{-2}$ while the fit $\chi^{2}=0.48$.

\footnotetext{
${ }^{1}$ For the energy range considered in our experiment the most important contribution originates from the $\rho$ meson.
} 


\section{Other channels.}

4.1 $\eta \rightarrow e^{+} e^{-}$

The $\eta \rightarrow e^{+} e^{-}$is a very rare two-body decay. The most recent theoretical calculations of this decay branching ratio provide values around $10^{-9}$. They use different models, among other ChPT [12], modified VMD, hidden gauge [13] and take into account various corrections (such as mass correction [14]).

In 2008, a branching ratio upper limit of $2.7 \cdot 10^{-5}$ at $90 \%$ C.L. was established for this channel in WASA-at-CELSIUS experiment and published in [16]. WASA-at-CELSIUS set-up consisted in the same WASA detector we use in this work while it operated at the CELSIUS storage ring located in Uppsala (Sweden) at The Svedberg Laboratory. This limit was extracted from a sample of $2.41 \cdot 10^{5} \eta$ mesons produced in $\mathrm{pd} \rightarrow{ }^{3} \mathrm{He} \eta$ reaction at $893 \mathrm{MeV}$ incident proton energy (close to $\eta$ production threshold).

The best actual upper limit is $<4.9 \cdot 10^{-6}$ (at $90 \%$ confidence level) and it was established by HADES experiment in 2012 [15]. HADES operates at GSI research centre in Darmstadt (Germany). This result was based on data collected in pp collisions at $3.5 \mathrm{GeV}$.

Up till now, there are only upper limits for $\eta \rightarrow e^{+} e^{-}$decay but if one were to determine a $\mathrm{BR}\left(\eta \rightarrow e^{+} e^{-}\right)$value exceeding $\sim 6 \cdot 10^{-9}$ it might be seen as a signature of physics beyond the SM.

The ongoing analysis on the new data set from WASA-at-COSY indicates a preliminary new limit on the $\operatorname{BR}\left(\eta \rightarrow e^{+} e^{-}\right)$of the order of $4 \cdot 10^{-6}$.

$4.2 \eta \rightarrow \pi^{0} e^{+} e^{-}$

The C-conserving reaction $\eta \rightarrow \pi^{0} \gamma^{*} \gamma^{*} \rightarrow \pi^{0} e^{+} e^{-}$is allowed in the SM but its theoretical $\mathrm{BR} \sim 10^{-9}-10^{-12}$. The decay such as $\eta \rightarrow \pi^{0} \gamma^{*} \rightarrow \pi^{0} e^{+} e^{-}$would violate C-parity. The current experimental limit on the $\operatorname{BR}\left(\eta \rightarrow \pi^{0} e^{+} e^{-}\right)<4 \cdot 10^{-5}$ (at $90 \%$ C.L.) thus there are four orders of magnitude between the experimental limit and the SM expectation. This means that there is still room for physics beyond the SM. Based on our new data from WASA-at-COSY, we can set a preliminary limit (for $e^{+} e^{-}$invariant masses larger than $100 \mathrm{MeV} / \mathrm{c}^{2}$ ) of about $2 \cdot 10^{-5}$ (at $90 \%$ C.L.).

\section{References}

[1] Adriani, Oscar et al., An anomalous positron abundance in cosmic rays with energies 1.5-100 GeV, Nature Vol.458:607-609, 2009

[2] Aharonian, F. et al., The energy spectrum of cosmic-ray electrons at TeV energies, Phys. Rev. Lett. Vol.101, 2008

[3] Chang, J. et al, An excess of cosmic ray electrons at energies of 300-800 GeV, Nature Vol.456:362-365, 2008

[4] Jean, Pierre et al, Early SPI / INTEGRAL measurements of $511 \mathrm{keV}$ line emission from the 4th quadrant of the Galaxy, Astron. Astrophys. Vol.407, 2003 
[5] Vecchi, Manuela, Precision measurement of the $\left(e^{+}+e^{-}\right)$flux in primary cosmic rays from $0.5 \mathrm{GeV}$ to $1 \mathrm{TeV}$ with the Alpha Magnetic Spectrometer on the International Space Station, 25th European Cosmic Ray Symposium (ECRS 2016) Turin, Italy, September 04-09, 2016

[6] Fayet, Pierre, Effects of the Spin 1 Partner of the Goldstino (Gravitino) on Neutral Current Phenomenology, Phys. Lett. Vol.B95, 1980

[7] Dobroliubov, M. I. et al, Neutral Pion Radiative Decay as an Efficient Tool for Searching for New Light Gauge Bosons, Phys. Lett. Vol.B206, 1988

[8] Boehm, C. and Fayet, P., Scalar dark matter candidates, Nucl. Phys. Vol.B683:217-263, 2004

[9] Alexander, J. et al, Dark Sectors 2016 Workshop: Community Report, http://inspirehep.net/record/1484628/files/arXiv:1608.08632.pdf, 2016

[10] Adlarson, P. et al., Search for a dark photon in the $\pi^{0} \rightarrow e^{+} e^{-} \gamma$ decay, Phys. Lett. Vol.B726:187-193, 2013

[11] Landsberg, L.G., Electromagnetic decays of light mesons, Phys. Rept. Vol.128:301-376, 1985

[12] Savage, M.J., The Rare decays $\pi^{0} \rightarrow e^{+} e^{-}, \eta \rightarrow e^{+} e^{-}$and $\eta \rightarrow \mu^{+} \mu^{-}$in chiral perturbation theory, Phys. Lett. Vol.B291:481-483, 1985

[13] Petri, T., Anomalous decays of pseudoscalar mesons, $\mathrm{PhD}$ thesis, Institut für Kernphysik Forschungszentrum, Jülich, Germany, 2010

[14] Dorokhov, A.E. et al, Complete structure dependent analysis of the decay $P \rightarrow l^{+} l^{-}$, Phys. Lett. Vol.B677:145-149, 2009

[15] Agakishiev, G. et al, Inclusive dielectron spectra in $p+p$ collisions at $3.5 \mathrm{GeV}$, Eur. Phys. J. Vol.A48, 2012

[16] Berlowski, M. et al, Measurement of eta meson decays into lepton-antilepton pairs, Phys. Rev. Vol.D77, 2008 